\title{
An analysis of the space-debris-deorbit process based on a mathematical model
}

\author{
Pengyu Zhao ${ }^{1, \text { a }}$, Mingxi Chen ${ }^{2, \mathrm{~b}}$ and Hongyu Fang ${ }^{3, \mathrm{c},{ }^{*}}$ \\ ${ }^{1}$ School of Materials Science and Engineering, Central South University, Changsha 410083, China; \\ ${ }^{2}$ School of Information Science and Engineering, Central South University, Changsha 410083, \\ China; \\ ${ }^{3}$ School of Mechanical and Electrical Engineering, Central South University, Changsha 410083, \\ China. \\ azhao_pengyu@163.com, bmingxi8chen@sohu.com, ${ }^{\mathrm{c}} 273462134 @ q q . c o m$ \\ Corresponding Author: Hongyu Fang
}

Keywords: space debris, mathematical model, deorbit, orbit location, feedback system.

\begin{abstract}
The relevant increase in the number of uncontrolled space debris is nowadays considered one of the main threats for future sustainability of space activities and space access. With the increase amount of space debris, space debris causes more widespread attention over the world. After analyzing the relationship between maneuvering energy, orbital transfer time and the orbital radius, a mathematical model has been established which can explain the mechanism of the space debris deorbit and improve the accuracy of orbit location. At the same time, a feedback decision-making system is proposed for choosing the best way to deorbit space debris though the analysis of the factors on the total cost.
\end{abstract}

\section{Introduction}

It is nowadays clearly that large amount of space debris in the vicinity of the earth have seriously threatened the future sustainability of space activities and space access [1]. Space debris is a specific type of space objects, also called orbital debris, that is human-made, no longer functional, and in Earth's orbit. Space debris mainly comes from dead spacecraft, lost equipment, boosters and weapons, which ranges in mass from several grams to many tons, and in diameter from a few millimeters to tens of meters. Due to their high orbital velocity in the orbit, even small-size debris can lead to catastrophic breakups, which has the effect of not only damaging operational spacecraft but also increasing the amount of hazardous debris, which in turn cause further space collisions with the rockets and spacecraft on a mission in orbit. The number of total objects orbiting around the earth grew dramatically over the years, with a clear acceleration since 2006. The growing number of debris is raising the probabilities of collision between two man-made space objects. The issue itself became more widely discussed in the news media when the Russian satellite Cosmos - 2251 and the USA satellite Iridium - 33 collided on 10 February, 2009 [2].

In the past few years, a number of different methods of removing the debris have been proposed, such as conductive tether wire performing electron collection from the ambient space plasma [3, 4] and high energy lasers used to target specific pieces of debris [5] and small satellites with robot arms designed to remove the debris [6, 7]. Each method may represent a valuable solution for space debris belonging to specific classes or types or orbiting in particular space regions. Nowadays, based on the advanced technology, the contact can be easily set up between the observation point on ground and debris in orbit through their azimuth and altitude angle to locate the debris. However, the change of the debris orbit due to some special and detail factors undoubtedly have an effect on the location accuracy. Therefore, to clean the near-Earth space from the dangerous debris more effectively, it is necessary to set up a mathematical model which can explain the mechanism of the space debris deorbit and improve the accuracy of orbit location. 


\section{Model}

A model was set up to explain how the debris escaped from the initial orbit. Here, taking into consideration that the debris is limited by the small size but still moves in low earth orbit. A kind of satellite laser can remove the debris to another orbit in current technological level. Two indexes were used to evaluate the clean-up operation based on orbit transferring. $\Delta V$ is the energy which can help the debris achieve track changes. $t$ indicates the time consumption in the process of orbital transfer. To simplify the model, the shape of the initial orbit is taken as being nearly circular and the transfer orbit happens only one time. $n_{T}$ is defined as the ratio between $r_{2}$ and $r_{1}$, namely, $n_{T}=\frac{r_{2}}{r_{1}}$, where $r_{2}$ and $r_{1}$ are the radius of the orbit after and before changing respectively, The expressions [8] are shown as the following:

$$
\begin{aligned}
& \Delta V=n_{T} \cdot v_{c} \sqrt{\frac{2}{n_{T}+1}} \\
& v_{c}=\sqrt{\frac{G \cdot m_{e}}{r_{1}}}
\end{aligned}
$$

Where $m_{e}$ means the mass of the Earth, $v_{c}$ means the speed in initial orbit.

$$
t=t_{p}-\pi \sqrt{\frac{\left(1+n_{T}\right)^{3}}{8 \mu r_{1}^{3}}}
$$

In which $t_{p}$ means the mission time, $\mu$ is a constant.

\section{Discussion}

As the above deduction shows, $\Delta V$ depends on the ratio between $r_{2}$ and $r_{1}$ when $n_{T}$ is treated as a constant, Then we have the following qualitative analysis conclusions:

$$
\begin{aligned}
& \Delta V \propto \frac{1}{\sqrt{r_{1}}} \\
& t \propto \frac{1}{\sqrt{r_{1}^{3}}}
\end{aligned}
$$

(5)

More daringly, we choose:

$$
\begin{aligned}
& \Delta V=\frac{1}{\sqrt{r_{1}}} \\
& t=\frac{1}{\sqrt{r_{1}^{3}}}
\end{aligned}
$$



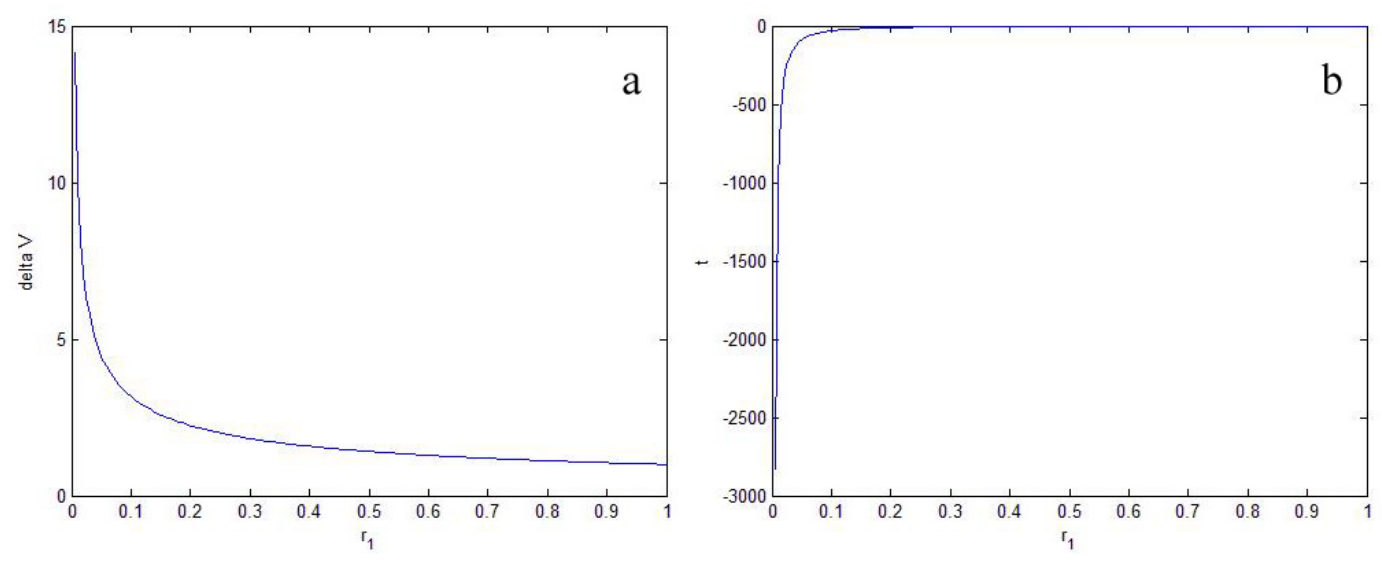

Fig. 1 a. The curve of $\Delta V$ and initial radius $r_{1}$; b. The curve of $t$ and initial radius $r_{1}$

Supposing $n_{T}$ remains unchanged, as initial radius increases, less energy is needed to remove the debris, but the total time increases. (Fig.1)

We also have

$\Delta V \propto \frac{n_{T}}{\sqrt{n_{T}+1}}$
$t \propto-\sqrt{\left(1+n_{T}\right)^{3}}$

Similarly, we choose:

$$
\begin{aligned}
& \Delta V=\frac{n_{T}}{\sqrt{n_{T}+1}} \\
& t=-\sqrt{\left(1+n_{T}\right)^{3}}
\end{aligned}
$$
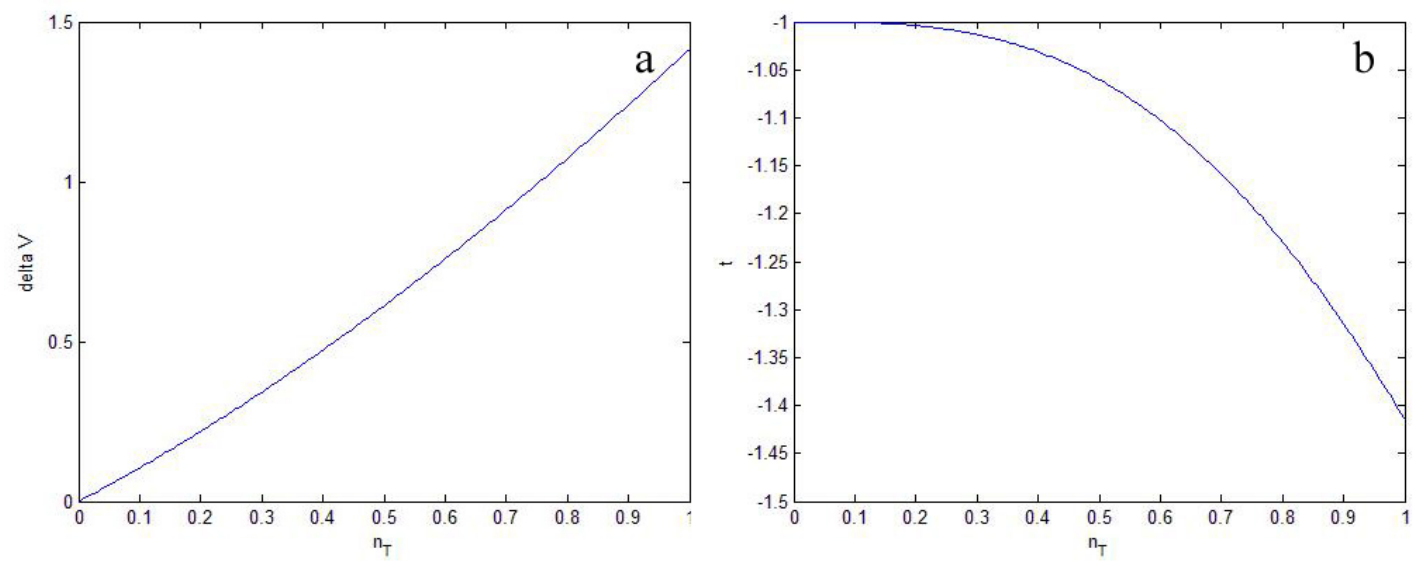

Fig. 2 a. The curve of $\Delta V$ and initial radius $n_{T}$; b. The curve of $t$ and initial radius $n_{T}$

Supposing $r_{1}$ remains unchanged, as $n_{T}$ increases, more energy is needed to remove the debris, but the total time decreases. (Fig.2)

\section{Solving}

$\Delta V$ and $t$ represent the cost of expenditure and effect respectively. Define $P$ as the total cost, which depends on $\Delta V$ and $t$. It can be described as the following equation.

$$
P=A \bullet \Delta V+B \bullet t
$$

Where $A$ and $B$ are the weight value of $\Delta V$ and $t$ respectively. $A$ and $B$ link tightly with the detailed method that is chosen to deorbit the debris, which should be assigned according to the actual circumstances by proper weight assignment methods. 
As the model shows, $\Delta V$ and $t$ are the function of $r_{2}$ and $r_{1}$. The equation could be specifically described by the usage of those two main indicators. After the simplified treatment, we can get,

$$
P=A \cdot \frac{r_{2}}{r_{1}} \cdot \sqrt{\frac{2 G m_{e}}{r_{2}+r_{1}}}+B \cdot\left(t_{P}-\frac{\pi\left(r_{1}+r_{2}\right)}{r_{1}} \cdot \sqrt{\left.\frac{r_{1}+r_{2}}{8 \mu}\right)}\right.
$$

According to the discussion, the effect on $P$ of $r_{2}$ and $r_{1}$ varies with the value and ratio. When the radius before and after the debris deorbit is determined, most of the parameters will be assured. Therefore, the total cost depends on the way which is chosen.

This model could be converted into codes and performed by computers, which could form a

\section{best way to deorbit the debris}

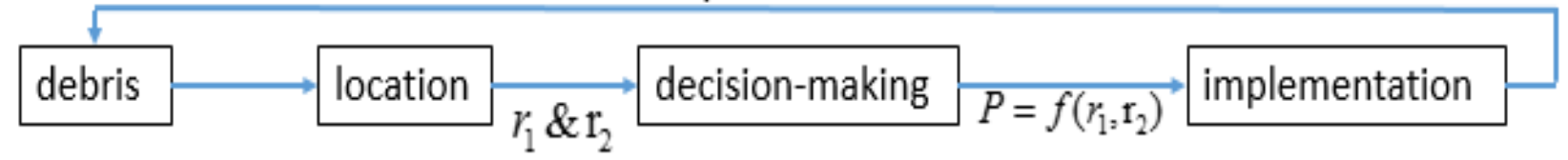

feedback system. (Fig. 3)

Fig. 3 decision-making system for the best way to deorbit the debris

\section{Summary}

In this paper, we establish a space-debris-deorbit model which can explain how the radiuses before and after obit changing affect the energy and time which is needed for removing the debris. After analyzing the model, we get the following conclusions: When the ratio between $r_{2}$ and $r_{1}$ remains unchanged, as initial radius increases, we need less energy to remove the debris, but the total time increases. When the initial radius remains unchanged, as the ratio between $r_{2}$ and $r_{1}$ increases, we need more energy to remove the debris, but the total time decreases.

As time and energy link tightly with cost which varies with the specific circumstances, we set up another model about the total cost of deorbiting space debris during the solving process. Through the analysis of the cost model, a feedback system for choosing the best way to deorbit the debris is proposed. Most of the parameters which may affect the cost could be assured by measuring the location of the space debris. Thus, the total cost of the energy and time consumption depends on the way we choose. It is important to choose a reasonable way to clean up space debris.

\section{References}

[1]. A. Ruggiero, P. Pergola, M. Andrenucci, Small Electric Propulsion Platform for Active Space Debris Removal, IEEE TRANSACTIONS ON PLASMA SCIENCE, VOL. 43, NO. 12, 2015(12)

[2]. NASA: Orbital Debris Quarterly News. Vol. 15, no. 3,http://orbitaldebris.jsc.nasa.gov/newsletter/pdfs/ODQNv15i3.pdf, 2011.

[3]. S. Nishida, S. Kawamoto, F. Okawa, F. Terui, A. Nakajima, S. Kitamura, R\&D status of the active removal system for space debris, in: Proceedings of the International Astronautical Congress, IAC2005, Fukuoka, 2005.

[4]. S. Kawamoto, T. Makida, F. Sasaki, Y. Okawa, S. Nishida, Precise numerical simulations of electrodynamic tethers for an active debris removal system, Acta Astronautica 59 (2006) 139-148. 
[5]. Shen Shuangyan, Jin Xing, Chang Hao, Cleaning space debris with a space-based laser system[J], Chinese Journal of Aeronautics, (2014), 27(4):805-811.

[6]. S. Nishida, T. Yoshikawa, Space debris capture by joint compliance controlled robot, in: Proceedings of the Advanced Intelligent Mechatronics, AIM2003, Kobe, 2003.

[7]. Shin-Ichiro Nishida, Satomi Kawamoto, Yasushi Okawa, Fuyuto Terui, Shoji Kitamura, Space debris removal system using a small satellite[J], Acta Astronautica65(2009)95-102.

[8]. Wei Erhu, Yan Wei,Real-time monitoring system space junk to establish the feasibility analysis[J],Journal of Geomatics,2007(4). 